\title{
Design for Spinning Goniometer Automatic Direction Finding 1,2,3
}

\author{
W. J. Lindsay and D. S. Heim
}

(September 29, 1960)

\begin{abstract}
This paper discusses some aspects of instrumentation design in making a spinning goniometer radio direction finder essentially automatic in operation. Also discussed is the application of narrow band synchronous post detection filtering for improving the bearing sensitivity.
\end{abstract}

\section{Introduction}

In this paper various aspects of the problem of automating a spinning goniometer radio direction finder (hereafter abbreviated SGDF) are discussed. These considerations involve the handling of the directional information and do not concern other control operations such as tuning, switching, etc. Such a system is desirable for use as a research tool and to study the feasibility of applying various concepts operationally. Although the work was done on a AN/TRD-4 unit, the concepts may be applied to any SGDF. The availability of automatic operation does not preclude the operation of the equipment either partially or entirely in the usual manual fashion.

In a narrow aperture SGDF the simulation of a spun antenna is achieved by balanced amplitude modulation of the incoming wave at a rate set by the spin frequency of the goniometer. If sense voltage (from an omnidirectional antenna) is added to this, the effect is to restore the carrier component and we have a conventional amplitude modulated signal. These two modes of operation will be referred to as the DSB and the AM cases respectively. The AM wave may be expressed as:

$$
e(t)=E\left(1+k \cos \omega_{1} t\right) e^{j \omega_{0} t}
$$

Taking the real part:

$R e[e(t)]=E \cos \omega_{0} t+\frac{K E}{2}\left[\cos \left(\omega_{0}+\omega_{1}\right) t+\cos \left(\omega_{0}-\omega_{1}\right) t\right]$

The detected envelope of the AM wave is;

$$
e_{d}=\left[-\left(1+k \cos \omega_{1} t\right)\right]
$$

While the detected envelope of the DSB wave

1 Contribution from Cooley Electronics Laboratory, Electrical Engineering Dept., University of Michigan, Ann Arbor, Mich. This work was supported by the U. S. Army Signal Research and Development Laboratory under Contract No. DA $36-039$ sc 78283

2 The presentation of this paper was sponsored by the Office of Naval Research. Reproduction in part or in whole is permitted for any use of the United States Government.

${ }_{3}$ Paper presented at the Conference on Transmission Problems Related to High-Frequency Direction Finding, at UCLA, June 21-24, 1960.
(AM wave minus the carrier) is:

$$
e_{d}=\frac{-K_{1}}{\pi}\left[1-\frac{2}{1 \cdot 3} \cos 2 \omega_{1} t-\frac{2}{3.5} \cos 4 \omega_{1} t-\ldots\right]
$$

In the above expressions $\omega_{1}$ is the modulation frequency of the spinning goniometer while $\omega_{0}$ is the center frequency of the incoming radio frequency wave. It will be noted that in (2) the sideband frequencies are displaced from center frequency only by the spin rate of the goniometer, which in the present case is $30 \mathrm{c} / \mathrm{s}$; also the fact that in the detected envelope of the DSB wave only even harmonies of the modulation frequency appear, there is no fundamental component. The detected envelopes of both the DSB and the AM waves are shown in figure 1.

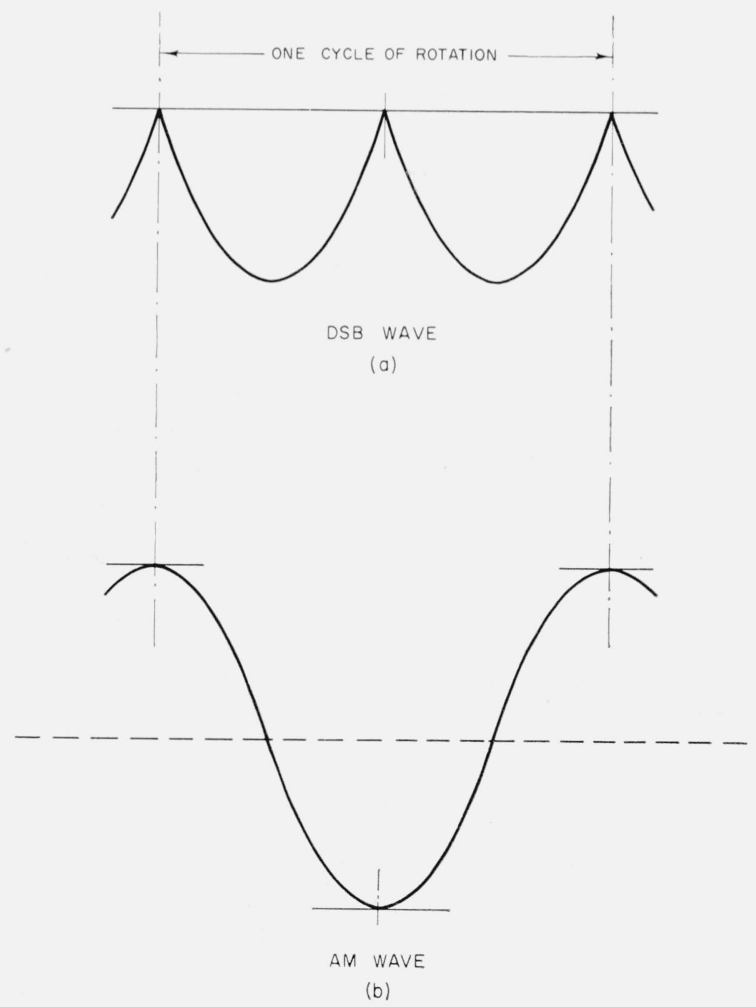

Figure 1. Waveforms for detected DSB and AM mode signals. 
In this type of system the bearings are determined by the position of the null of the antenna pattern with respect to a point of reference, usually North. It is seen that in figure 1a there are two such points, thus the familiar $180^{\circ}$ ambiguity, while in figure $1 \mathrm{~b}$ there is only one point at which the signal goes through the null in a specified direction of slope of the wave. It should be noted that the null point has been shifted by decoupling to the dotted line. This brings about an effect, which at present is the most significant detriment to the bearing accuracy obtained, of a shift in the apparent bearing when the character of the receiver is altered even slightly. For example when the gain control is changed over its range the bearing will change by two or three degrees. Since, as explained in a subsequent section, the reconstruction of the visual display and the zero crossing comparison for measuring relative phase both depend on having a constant average output from the receiver for a given input signal form but at different levels, it is evident that a receiver is needed that has low envelope distortion in order to eliminate this effect. There is indeed a great need for receivers designed for instrumentation purposes as well as communications, particularly with regard to the phase character of the receiver. If the signal is pulsed then the problem of maintaining the average is more difficult because $\mathrm{d}-\mathrm{c}$ components are introduced by the pulsing action of the signal. These will average out over a period of time, but for short periods cause havoc in the bearing determination. In viewing the DSB and AM situations certain things come to mind with regard to performing (1) Instrumental measurement of the bearing angle, and (2) Improvement of the signal to noise ratio by applying narrow band synchronous post detection filtering.

In the DSB case the null point region is that of the lowest signal to noise ratio in the entire cycle of rotation; thus in a noisy situation, which is the usual case rather than the exception, the null position is imperfectly defined, and indeed the whole structure of the wave, that is the collection of harmonies which define the structure, is needed in order to determine just where the average null point is located. A similar effect may be had by filtering out the second harmonic term in (4) and noting the relative phase of this wave to a reference wave.

In fact an increase in signal to noise ratio comparable to the AM case may be achieved but since an additional ambiguity is introduced this is not as attractive as using automatic sense if one wants to use narrow band filtering. If it is desired to keep the DSB information as is, except to filter the noise from it, then a comb filter may be used. This may be either the discrete frequency type or more likely the commutated type. In either case it is considerably more involved than a simple narrow-band single frequency filter. In the AM case the phase measurement is made on a sine wave (for a continuous signal) which has the same fundamental period as the goniometer rotation therefore the ambiguity is removed, and one may now apply filtering at the basic scan frequency rather than at twice the scan frequency. A description of the means of obtaining automatic sense, the design of the bearing readout system and a discussion of narrow band post detection filtering with some operational illustrations follow. An important aspect of the design is minimum cost with reasonable performance and simplicity.

\section{Automatic Sense}

The addition of a carrier or sense signal to the goniometer output changes the receiver input to a standard amplitude-modulated signal, modulated at the spin rate. The phase of this modulation relative to the phase of the rotation gives an unambiguous bearing. It can be shown that if the phase of the injected carrier is within plus or minus $90^{\circ}$ of the correct value it distorts the envelope, but only to the extent of adding harmonics (which may be filtered out) and causing a decrease in fundamental amplitude. It does not affect the relative time phase of the fundamental. Thus the generation of the sense signal is not so critical as is often supposed. If the sense signal is larger than the directional signal and is held to within $\pm 45^{\circ}$ of the proper value, adequate performance may be obtained.

The simplest means for obtaining the sense signal is to insert an antenna in the center of the DF array. Since the goniometer output has suffered some loss, as well as being the difference between two signals, the injected sense voltage can easily be made sufficiently large. If the distance across the array is small compared to a wavelength, a fairly simple network will maintain the phase margin within the required limits [Keen, 1947]. In fact, with rather heavy filtering, satisfactory operation has been obtained in the case illustrated with no additional phasing over the band of operation. The $\mathrm{R}-390$ receiver is conveniently designed with both a balanced and unbalanced input so that the sense signal may be fed directly into the unbalanced antenna input jack.

The instrumentation for producing the proper pattern on the screen of the CRT is depicted in figure 2. This unit serves two functions: (1) it

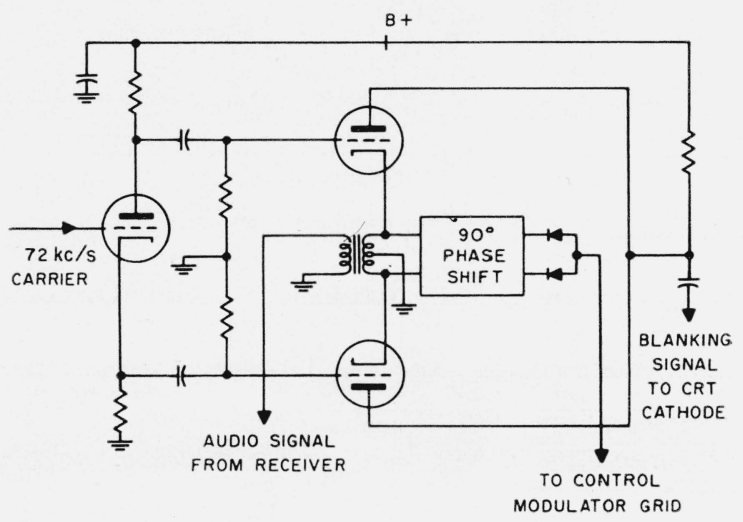

Figure 2. Automatic sense circuit. 
full-wave-rectifies the sine wave output of the receiver to give the usual propeller-type display, (2) it causes a blanking signal to be applied to the grid of the CRT in such a manner that only one half of the propeller appears on the screen. In the AN/TRD-4 equipment the display is filled in with a $72 \mathrm{kc} / \mathrm{s}$ carrier. Figure 3 shows the usual input to the indicator goniometer during one complete revolution during DSB type operation. It can be seen that the propeller is described on the face of the tube twice during each revolution. During period $T_{1}$ the tube must be blanked during the negative excursions of the $72 \mathrm{kc} / \mathrm{s}$ carrier. During period $T_{2}$, since the goniometer has rotated $180^{\circ}$, the blanking must occur on the positive excursions if the same half of the propeller is to be displayed. It can be seen from the circuit that with no signal from the receiver both triodes will conduct equally, and, since their inputs are $180^{\circ}$ out of phase, cancellation will occur in the plate circuit. In the presence of a signal from the receiver (ideally a sine wave), the tubes will alternately conduct fully and be cut off. It is this alternating operation which causes the proper balf of the display to slmays appear as discussed above. The resultant $72 \mathrm{kc} / \mathrm{s}$ signai, when annliad to the CRT grid, blanks during the negative excursions and brightens or enhances the desired half of the propeller during the positive excursions.

\section{Bearing Readout Computer}

The basis for the computer design is that of zerocrossing comparison, as has been used by others [Florman and Tait, 1949; Hatch and Bvatt, 1958]. Once the zero-crossing of the reference and DF signals have been determined they may be used to activate either an analog-type coincidence detector or a digital-type bearing computer. It was concluded that for visual monitoring purposes the analog was more suitable, and for detailed processing of the information the direct digital readout was more suitable. For the purpose at hand there was no need to eliminate the $180^{\circ}$ ambiguity from the analog readout.

The analog coincidence detector is fed into one channel of a two-channel Brush Recorder. The second channel is used to record signal strength. The particular Brush Recorder used has a frequency response up to about $50 \mathrm{c} / \mathrm{s}$. It is desirable to have an adjustable readout rate in the digital system so that the bearing readout rate can be adjusted to fit the study being made.

For studying the fast-fade variations, Bain [1955] indicates that in order to study the phenomena one should be able to take at least several bearings per second. Of course the rate for slow fluctuation studies should be much less than this, thus there exists the need for variable readout rate.

Reference is made to figure 4 , a block diagram of the readout system. The goniometer bearing scale was removed and machined out under the thumb rim, and $1^{\circ}$ slits were cut on the outer surface of the rim with an indexing head. Thus, with a light in the

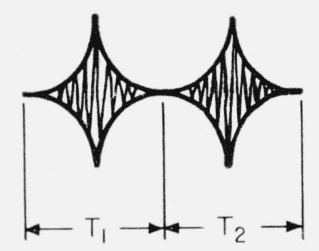

Figure 3. Input to display goniometer during conventional DSB operation.

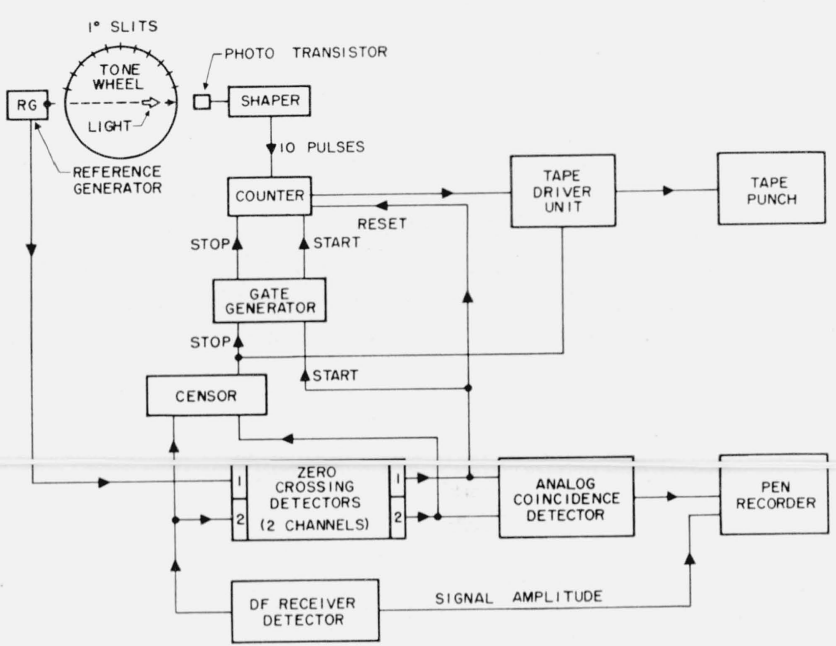

Figure 4. Block diagram of automatic bearing computer and readout.

wheel and a phototransistor on the outside, a tone wheel is available. A properly adjusted slit in front of the transistor is a necessity. The output of the phototransistor is fed into a two-stage transistor preamplifier and a pulse-forming circuit, then to the counter.

The reference generator is a small permanent-magnet-type alternator which was built on the end of the goniometer and is coupled directly to the shaft. The output from the reference generator is squared and formed into the start pulse in the zero-crossing detector. The output from the DF receiver is similarly treated in the zero-crossing detector unit. The squaring circuitry outputs from these units are fed to the analog coincidence detector and thence to the pen recorder for the visual record of bearing. The pulse output from the reference channelstarts the counter, and the pulse output from the receiver stops the counter. The censor unit at present is to be used as a yes-no stop pulse control depending on signal level. The start pulse is delayed slightly in the gate generator in order that the same signal may be used as a reset control at North position. When the censor unit says "no" then the counter will cycle on a $360^{\circ}$ count until the censor unit says "yes". The reference point, North in this case, may easily be moved to some other point around the compass if desired. The problem here is that of averaging through the cut point. For example, if the bearings 
were symmetrically distributed about North, than the average of these would be South, obviously the wrong answer. One simple solution to this is to have two cut points with two counts and pick the output that is farthest removed from a cut point. The counter output is then fed to the tape punch driver, which is controlled by the censored stop pulse, and thence punched onto paper tape. Since this unit is to be transistorized the Tally 8 channel punch was chosen. Eight channels allow a sector coverage of $255^{\circ}$. In order to cover the full $360^{\circ}$ sequential channel coding must be used which still allows a maximum of 30 readings per second.

\section{Narrow Band Synchronous Post Detection Filtering}

The application of NBSPD filtering has been discussed in the literature [Busignies and Dishal, 1949], however little seems to have been done to exploit its potential to improve bearing sensitivity. In fact, as Busignies and Dishal state, it appears that the capability of utilizing this is one of the fundamental advantages of this type of DF system over the co-operative navigational type system. This stems from the simple fact that in the great majority of situations the determination of the average direction of arrival over period even as short as just a few seconds is a very narrow band process. From an informational point of view the channel capacity required is in terms of bandwidth the approximate frequency of variation of the bearing one wishes to observe. This is of course determined exactly by the reading time of the specific receiving system in use. This means that for most purposes a bandwidth of a few cycles (say less then 10) will be adequate, unless there is some wideband information in addition to the bearing information, or if one is interested in studying the instantaneous rapid fluttering effects caused by wave interference, or for other specialized types of signals. There is more to be gained from a given factor of bandwidth reduction in the predetection circuitry than at post detection. By the same token this is much more difficult to achieve. In applying post detection narrowing if $\mathrm{BW}$ (IF) is the bandwidth of the IF in the receiver and $\mathrm{BW}(\mathrm{A})$ is the modified audio bandwidth then the following relation gives the input carrier ratio for equal output in terms of bearing determination. This is done for purposes of comparison to the operational illustration shown later.

$$
\text { Carrier Ratio }=\mathrm{K}\left[\frac{\mathrm{BW}(\mathrm{IF})}{\mathrm{BW}(\mathrm{A})}\right]^{1 / 4}
$$

and substituting for $\mathrm{BW}(\mathrm{IF})=2.0 \mathrm{kc}$

$$
\begin{gathered}
\mathrm{BW}(\mathrm{A})=5.0 \mathrm{c} / \mathrm{s} \\
\mathrm{CR}=\mathrm{K}\left[\frac{2000}{5}\right]^{1 / 4}=\mathrm{K}(400)^{1 / 4} \cong \mathrm{K}(4.475)
\end{gathered}
$$

This means a difference in terms of $\mathrm{db}$ of:

$$
\text { CR Diff }(\mathrm{db})=20 \log 10(4.475)
$$

$$
=13.0 \mathrm{db}
$$

Results from operation in this manner with automatic sense are given for purposes of illustration in figures 5 through 11 . A single tuned circuit with center frequency at the rotational frequency of the goniometer is placed after the detector. This unit has adjustable bandwidth with the narrowest position at about $5.0 \mathrm{c} / \mathrm{s}$. This is the condition under which the pictures were made. This gives a reading' time of about 140 msec. which allows observation of bearing changes up to about $7.0 \mathrm{c} / \mathrm{s}$.

Figure 5 shows a strong stable signal in the broadcast band, station WJR in Detroit. The double tip is due to imperfect foldover in the automatic sense circuit, which is being corrected.

In figure 6 , (a) and (b) are, respectively, the conventional TRD-4 display and the modified display, in which the target signal, at $6.93 \mathrm{Mc} / \mathrm{s}$, was adjusted in level until one could just estimate a bearing, at around $105^{\circ}$, on the conventional TRD-4 display. These shots are all 4 -sec time exposures.

In figure 7 , the signal level is reduced by $3.6 \mathrm{db}$, at which point the TRD-4 display is just barely discernible as a bearing indication.

In figure 8, the signal level is reduced from where it was in figure 6 , by $9.6 \mathrm{db}$, which leaves nothing in figure $8(\mathrm{a})$, although it can be seen that there is still quite a good bearing indication in figure $8(b)$. The limit is reached by reducing the signal level by another $6.0 \mathrm{db}$, producing figure 9. One can still get a fair estimate of the bearing, and the pattern which resembles a one-sided halo, is developing into

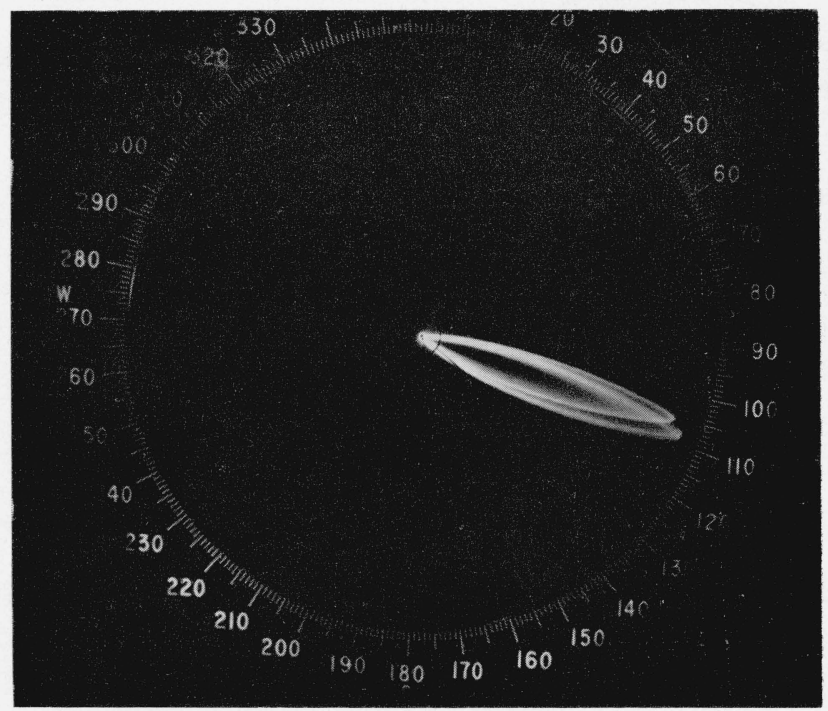

Figure 5. Bearing indication on modified AN/TRD-4 for strong stable signal. 


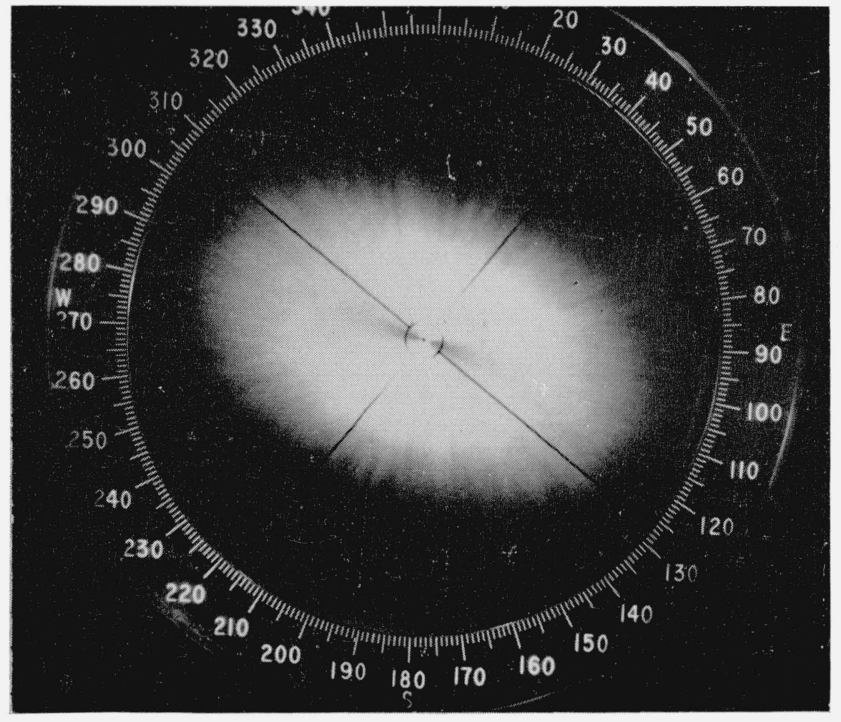

a

FiguRE 6. Comparison of operational results from (a) conventional AN/TRD-4 and (b) modified AN/TRD-4.

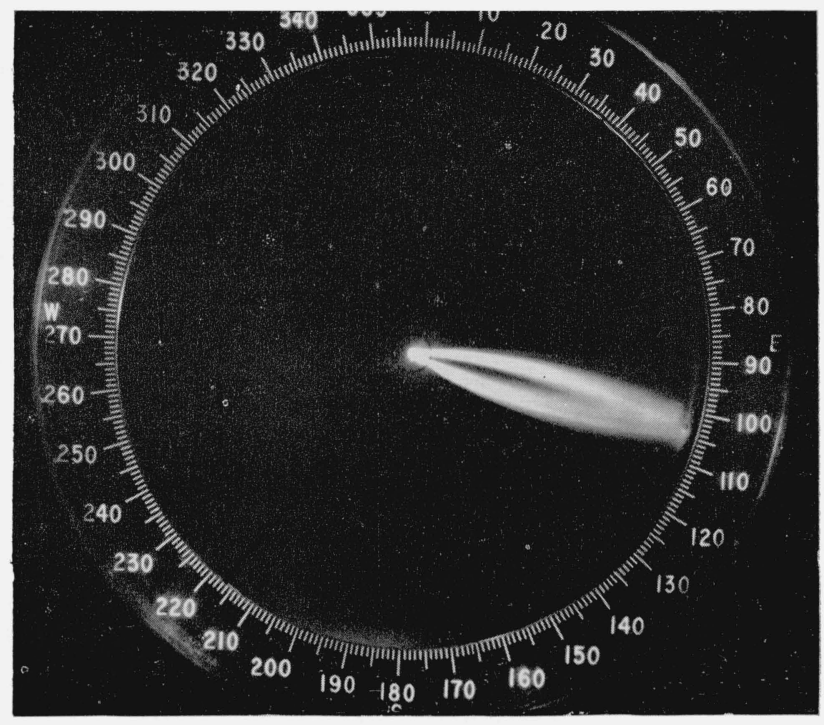

b

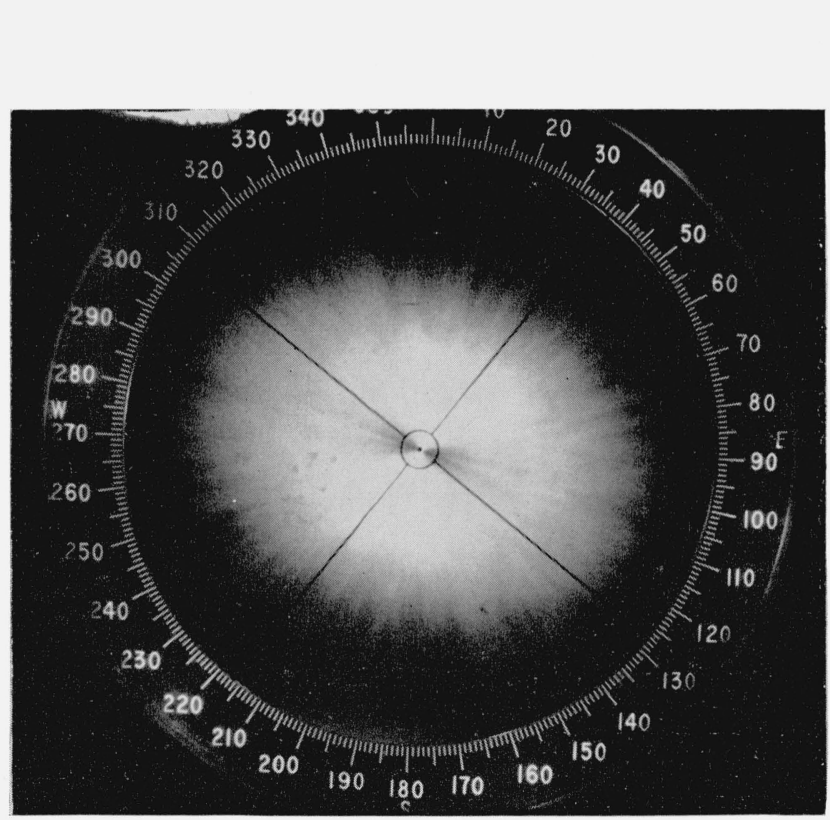

a

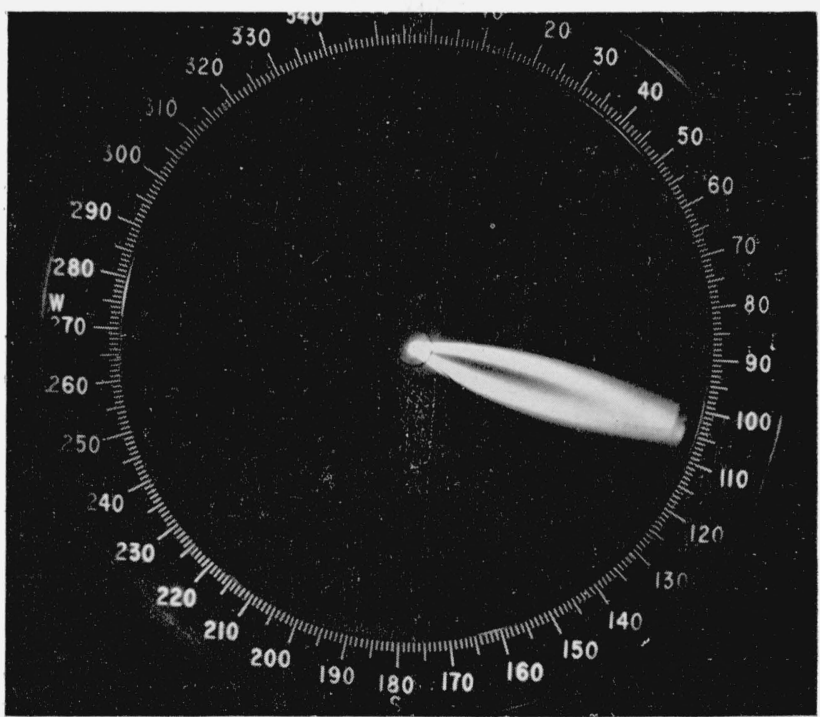

Figure 7. Comparison of operational results from (a) conventional AN/TRD-4 and (b) modified AN/TRD-4. Signal Level Reduced by $3.6 \mathrm{db}$ from that in figure 6 . 


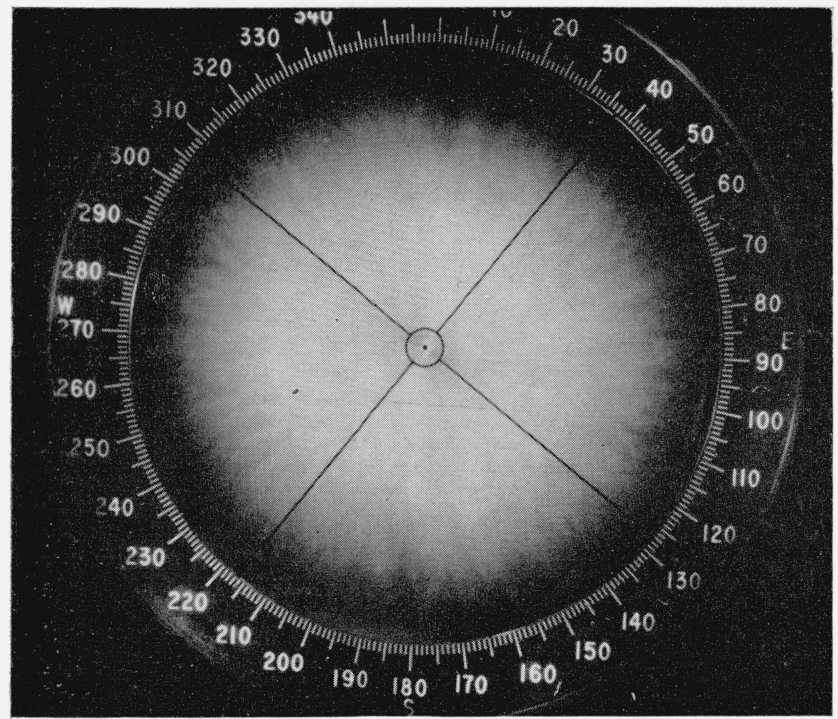

a

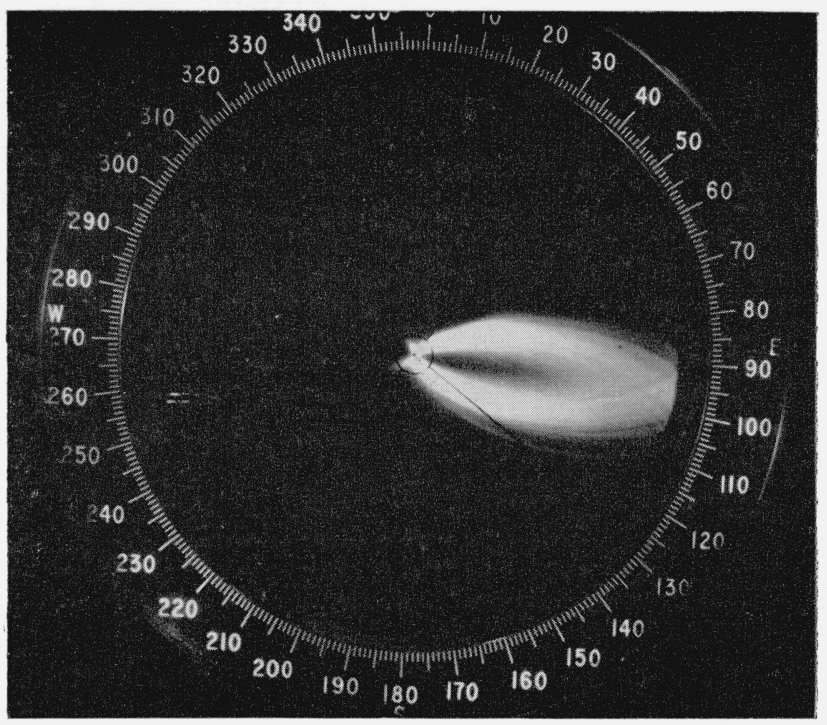

b

Figure 8. Comparison of operational results from (a) conventional AN/TRD-4 and (b) modified AN/TRD-4. Signal Level Reduced by $9.6 \mathrm{db}$ from that in figure 6 .

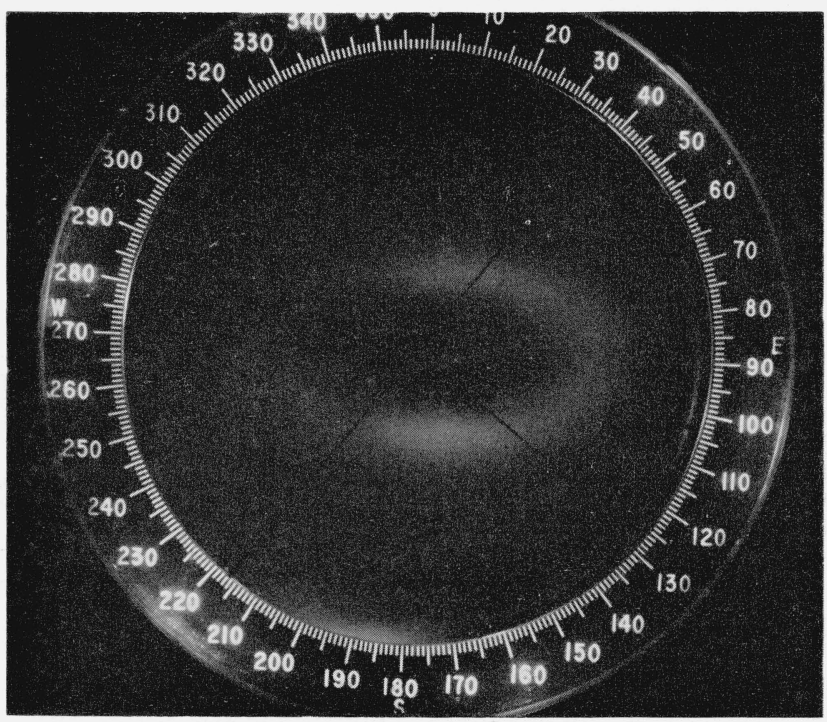

FIgURE 9. Threshold condition for the modified $A N / T R D-4$.

the characteristic background for this mode of operation. With no signal there is a faint green background level on the CR'T face. When a signal is tuned in the propeller half suddenly rises out of the hazy background as though by intensity modulation. The difference between the input signal level for figure 6 , and that of figure 9 , is $15.6 \mathrm{db}$.
Figure 10, depicts the appearance of a typical, fairly-weak FSK signal at $6.901 \mathrm{Mc} / \mathrm{s}$.

Figure 11, shows the target signal at $6.93 \mathrm{Mc} / \mathrm{s}$. being pulsed at $20 \mathrm{c} / \mathrm{s}$. The integration effect of the film favors 10 a more so than the eye in practice.

\section{Summary}

It has been demonstrated that one may partially automate and otherwise improve the operation of a SGDF with respect to certain prescribed criteria with rather simple and inexpensive methods. One must of course keep in mind what the particular objectives for the system are and the resulting advantages to be gained by making certain changes and the price that sometimes must be paid to make those changes.

\section{References}

Bain, W. C., On the rapidity of fluctuations in continuouswave radio bearings at high frequencies, Proc. Inst. Elec. Engrs., Pt. B, 102, 541-543 (July 1955).

Busignies, H., and M. Dishal, Some relations between speed of indication, bandwidth, and signal-to-random-noise ratio in radio navigation and direction finding, Proc. I.R.E. 37, No. 5, 478-488 (May 1949).

Florman, E. F., and A. Tait, An electronic phasemeter, Proc. I.R.E. 37, No. 2, 207-210 (February 1949).

Hatch, J. F., and D. W. G. Byatt, Improvements in HF direction finding by automatic time averaging, Marconi Review XXI, No. 128, 16-29 (1958)

Keen, R., Wireless direction finding, pp. 144-149 (Ilife and Sons, Ltd., London, 1947). 


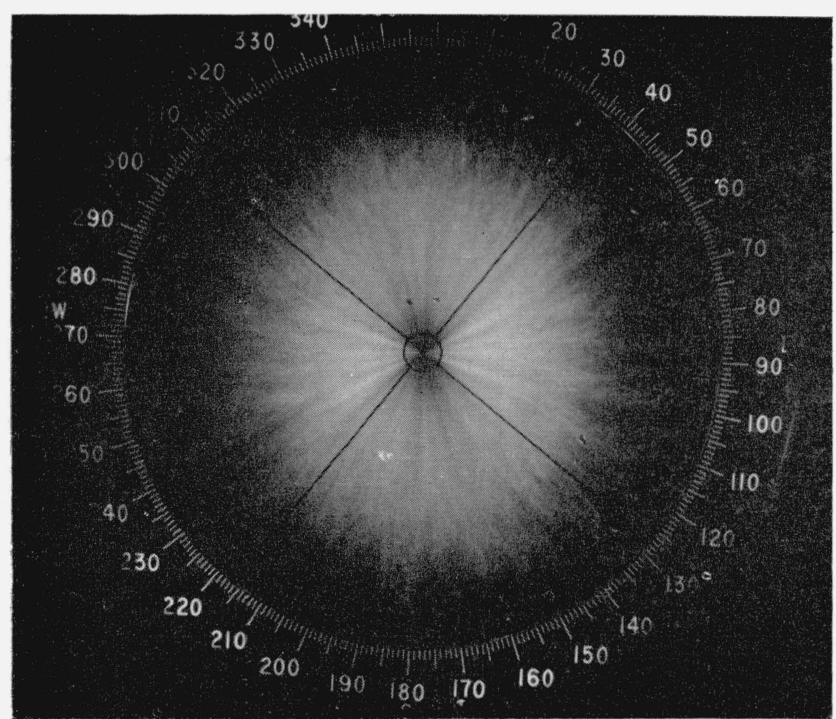

a

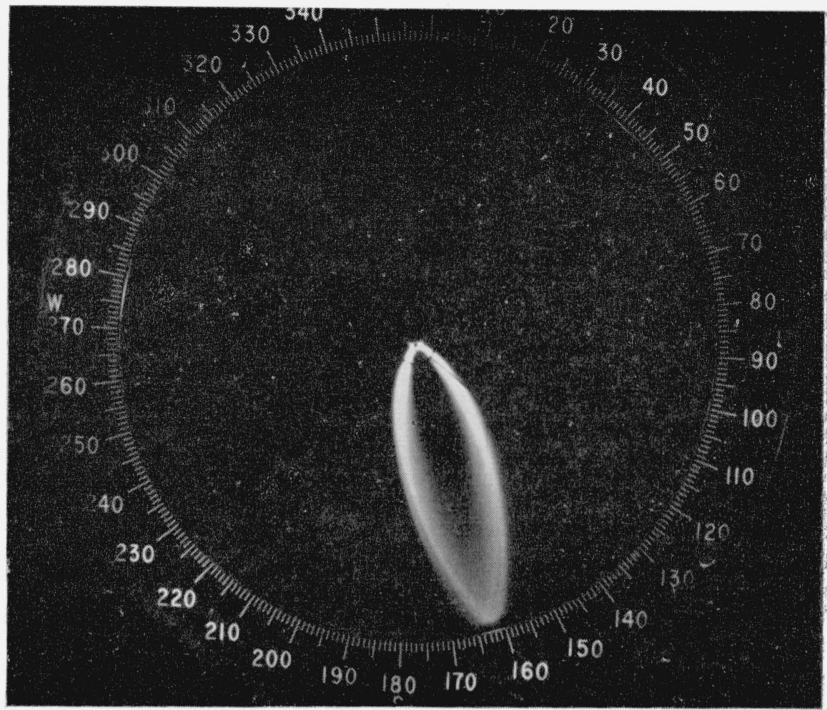

b

Figure 10. Typical, weak FSK signal, as obtained from (a) conventional AN/TRD-4 and (b) modified AN/TRD-4.

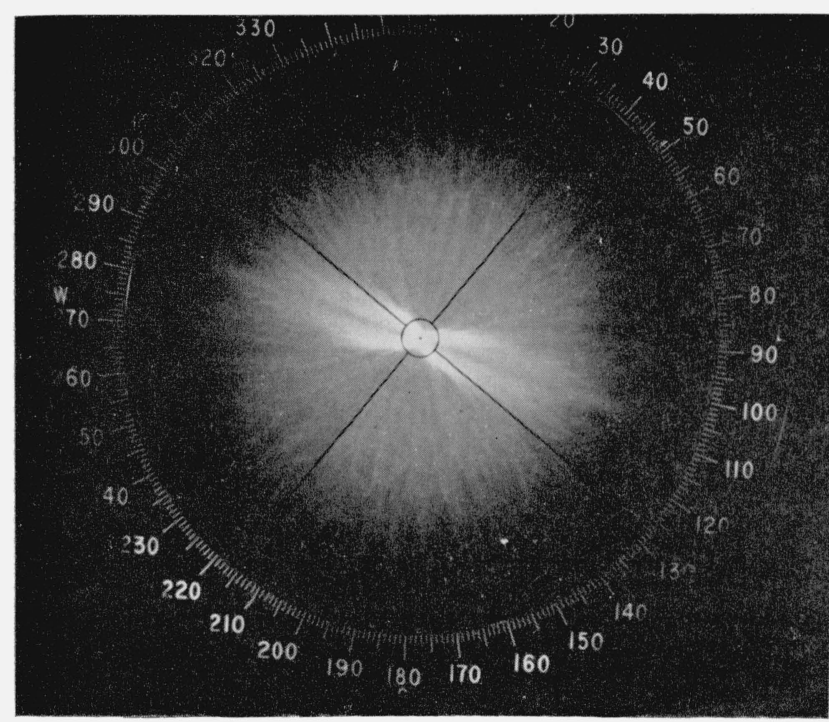

a

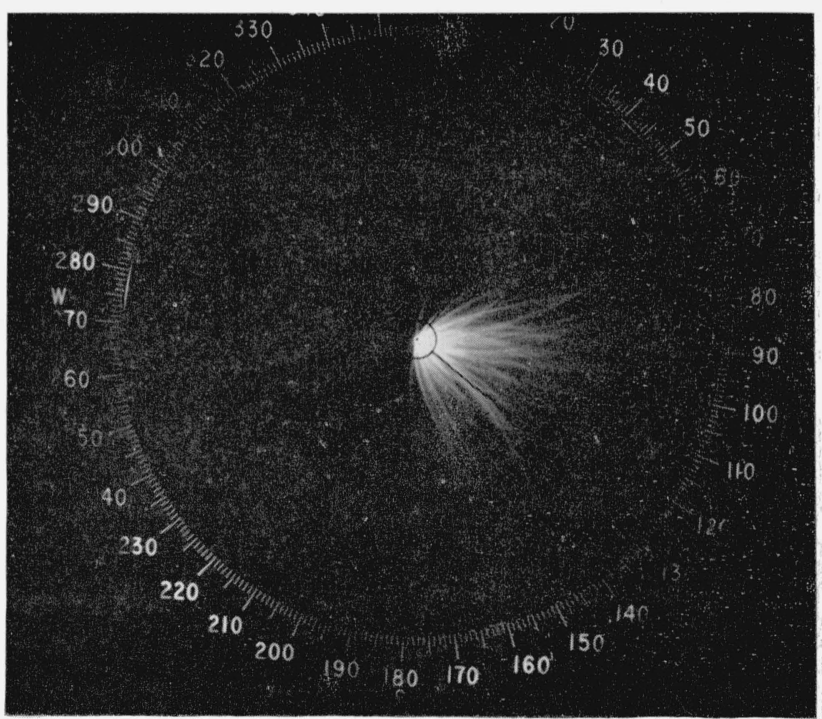

b

Figure 11. Pulsed signal, as sbtained from (a) conventional AN/TRD-4 and $(b) \operatorname{modified~} A N / T R D-4$.

(Paper 65D3-125) 